\title{
Role Conjunctions in Expressive Description Logics
}

\author{
Birte Glimm and Yevgeny Kazakov \\ Oxford University Computing Laboratory
}

\begin{abstract}
We show that adding role conjunctions to the Description Logics (DLs) $\mathcal{S H \mathcal { I }}$ and $\mathcal{S H O \mathcal { F }}$ causes a jump in the computational complexity of the standard reasoning tasks from ExpTime-complete to 2ExpTime-complete and from NExpTime-complete to N2ExpTime-hard respectively. We further show that this increase is due to a subtle interaction between inverse roles, role hierarchies, and role transitivity in the

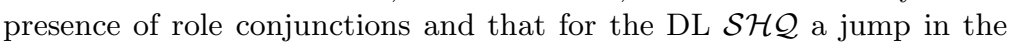
computational complexity cannot be observed.
\end{abstract}

\section{Introduction}

Description Logics are knowledge representation formalisms [1], which are mostly based on decidable fragments of First-Order Logic with only unary and binary predicates, called concepts and roles. The DLs $\mathcal{S H \mathcal { I } F}$ and $\mathcal{S H O I N}$ provide a logical underpinning for the W3C standards OWL Lite and OWL DL and highly optimized reasoner implementations are available.

Current standardization efforts go into the direction of also supporting a richer set of constructors for roles. It was recently shown that role compositions in the proposed OWL2 standard cause an exponential blowup [2] in the computational complexity of the standard reasoning problems. We show that allowing for conjunctions over roles can also cause such a blowup.

Role conjunctions are closely related to conjunctive queries. In [3] it was shown how the problem of conjunctive query answering over $\mathcal{S H I Q}$ can be reduced to reasoning in $\mathcal{S H I} \mathcal{Q}^{\sqcap}$ - the extension of $\mathcal{S H \mathcal { I } Q}$ with role conjunctions. For example, the query $\langle x\rangle \leftarrow r(x, y) \wedge s(x, y) \wedge A(y)$ can be answered by retrieving all instances of the concept $\exists(r \sqcap s)$. $A$ for $A$ a concept name, $r, s$ roles, and $x, y$ variables. In [3] it was also shown that reasoning in $\mathcal{S H I \mathcal { I }}{ }^{\sqcap}$ is in 2 ExpTime. It was an open question whether this bound is tight.

In this paper we demonstrate that standard reasoning in $\mathcal{S H I \mathcal { I }} \sqcap$ and even in $\mathcal{S H} \mathcal{I}^{\sqcap}$ is 2 ExpTime-hard. It follows from the construction in [3] that reasoning in $\mathcal{S H I} \mathcal{Q}^{\sqcap}$ is in ExpTime when either the number of transitive roles in role inclusions, or the length of role conjunctions is bounded. We also demonstrate that reasoning in $\mathcal{S H \mathcal { I }} \mathcal{Q}^{\sqcap}$ without inverse roles is in ExpTime as well. Thus, the increased complexity of $\mathcal{S H I} \mathcal{Q}^{\sqcap}$ is due to a combination of inverse roles, role transitivity, role hierarchies, and role conjunctions. A similar effect is observed for propositional dynamic logics (PDL), where the intersection operator causes 
a complexity jump from ExpTime to 2ExpTime [4]. PDL is closely related to the DL $\mathcal{A L C}$ extended with regular expressions on roles.

We now introduce some basic definitions and notations used throughout the paper. In Section 3, we prove that for $\mathcal{S H} \mathcal{Q}^{\sqcap}$ the standard reasoning tasks remain in ExpTime. In Section 4, we present the 2ExpTime-hardness result for $\mathcal{S H} \mathcal{H} \mathcal{I}^{\sqcap}$ by a reduction to the word problem for exponentially space bounded Turing

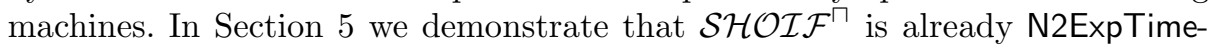
hard using a reduction to domino tiling problems. This paper is accompanied by a technical report which contains intermediate lemmata and full proofs [5].

\section{Preliminaries}

Let $N_{C}, N_{R}$, and $N_{I}$ be countably infinite sets of concept names, role names, and individual names. We assume that the set of role names contains a subset $N_{t R} \subseteq N_{R}$ of transitive role names. A role $R$ is an element of $N_{R} \cup\left\{r^{-} \mid r \in N_{R}\right\}$, where roles of the form $r^{-}$are called inverse roles. A role conjunction is an expression of the form $\rho=\left(R_{1} \sqcap \cdots \sqcap R_{n}\right)$. A role inclusion axiom (RIA) is an axiom of the form $R \sqsubseteq S$ where $R$ and $S$ are roles. A role hierarchy $\mathcal{R}$ is a finite set of role inclusion axioms.

An interpretation $\mathcal{I}=\left(\Delta^{\mathcal{I}},{ }^{\mathcal{I}}\right)$ consists of a non-empty set $\Delta^{\mathcal{I}}$, the domain of $\mathcal{I}$, and a function ${ }^{\mathcal{I}}$, which maps every concept name $A$ to a subset $A^{\mathcal{I}} \subseteq \Delta^{\mathcal{I}}$, every role name $r \in N_{R}$ to a binary relation $r^{\mathcal{I}} \subseteq \Delta^{\mathcal{I}} \times \Delta^{\mathcal{I}}$, every role name $r \in N_{t R}$ to a transitive binary relation $r^{\mathcal{I}} \subseteq \Delta^{\mathcal{I}} \times \Delta^{\mathcal{I}}$, and every individual name $a$ to an element $a^{\mathcal{I}} \in \Delta^{\mathcal{I}}$. The interpretation of an inverse role $r^{-}$is $\left\{\left\langle d, d^{\prime}\right\rangle \mid\left\langle d^{\prime}, d\right\rangle \in r^{\mathcal{I}}\right\}$. The interpretation of a role conjunction $R_{1} \sqcap \cdots \sqcap R_{n}$ is $R_{1}^{\mathcal{I}} \cap \cdots \cap R_{n}^{\mathcal{I}}$. An interpretation $\mathcal{I}$ satisfies a RIA $R \sqsubseteq S$ if $R^{\mathcal{I}} \subseteq S^{\mathcal{I}}$, and a role hierarchy $\mathcal{R}$ if $\mathcal{I}$ satisfies all RIAs in $\mathcal{R}$.

For a role hierarchy $\mathcal{R}$, we introduce the following standard DL notations:

1. We define the function $\operatorname{Inv}$ over roles $\operatorname{as} \operatorname{Inv}(r):=r^{-}$and $\operatorname{lnv}\left(r^{-}\right):=r$ for $r \in N_{R}$.

2. We define $\sqsubseteq_{\mathcal{R}}$ as the smallest transitive reflexive relation on roles such that $R \sqsubseteq S \in \mathcal{R}$ implies $R \sqsubseteq_{\mathcal{R}} S$ and $\operatorname{lnv}(R) \sqsubseteq_{\mathcal{R}} \operatorname{lnv}(S)$. We write $R \equiv_{\mathcal{R}} S$ if $R \sqsubseteq_{\mathcal{R}} S$ and $S \sqsubseteq_{\mathcal{R}} R$.

3. A role $R$ is called transitive w.r.t. $\mathcal{R}$ (notation $R^{+} \sqsubseteq_{\mathcal{R}} R$ ) if $R \equiv_{\mathcal{R}} S$ for some role $S$ such that $S \in N_{t R}$ or $\operatorname{lnv}(S) \in N_{t R}$.

4. A role $S$ is called simple w.r.t. $\mathcal{R}$ if there is no role $R$ such that $R$ is transitive w.r.t. $\mathcal{R}$ and $R \sqsubseteq_{\mathcal{R}} S$. A role conjunction $R_{1} \sqcap \cdots \sqcap R_{n}$ is simple w.r.t. $\mathcal{R}$ if each conjunct is simple w.r.t. $\mathcal{R}$.

The set of $\mathcal{S H O I} \mathcal{Q}^{\sqcap}$-concepts is the smallest set built inductively from $N_{C}, N_{R}$, and $N_{I}$ using the following grammar, where $A \in N_{C}, o \in N_{I}, n$ is a non-negative integer, $\rho$ is a role conjunction and $\delta$ is a simple role conjunction:

$$
C::=A|\{o\}| \neg C\left|C_{1} \sqcap C_{2}\right| \forall \rho . C \mid \geqslant n \delta . C .
$$


We use the following standard abbreviations: $C_{1} \sqcup C_{2} \equiv \neg\left(\neg C_{1} \sqcap \neg C_{2}\right), \exists \rho . C \equiv$ $\neg(\forall \rho .(\neg C))$, and $\leqslant n \delta . C \equiv \neg(\geqslant(n+1) \delta . C)$.

The interpretation of concepts in $\mathcal{I}$ is defined as follows:

$$
\begin{aligned}
& \{o\}^{\mathcal{I}}=\left\{o^{\mathcal{I}}\right\},(C \sqcap D)^{\mathcal{I}}=C^{\mathcal{I}} \cap D^{\mathcal{I}},(\neg C)^{\mathcal{I}}=\Delta^{\mathcal{I}} \backslash C^{\mathcal{I}}, \\
& \quad(\forall \rho . C)^{\mathcal{I}}=\left\{d \in \Delta^{\mathcal{I}} \mid \text { if }\left\langle d, d^{\prime}\right\rangle \in \rho^{\mathcal{I}}, \text { then } d^{\prime} \in C^{\mathcal{I}}\right\}, \\
& (\geqslant n \delta . C)^{\mathcal{I}}=\left\{d \in \Delta^{\mathcal{I}} \mid \sharp s^{\mathcal{I}}(d, C) \geq n\right\}
\end{aligned}
$$

where $\sharp M$ denotes the cardinality of the set $M$ and $s^{\mathcal{I}}(d, C)$ is defined as $\left\{d^{\prime} \in\right.$ $\Delta^{\mathcal{I}} \mid\left\langle d, d^{\prime}\right\rangle \in s^{\mathcal{I}}$ and $\left.d^{\prime} \in C^{\mathcal{I}}\right\}$. Concepts of the form $\{o\}$ are called nominals.

The DL $\mathcal{S H O \mathcal { H }} \sqcap$ is obtained by only allowing for the declaration of roles as functional (e.g., Func $(R)$ ) instead of full number restrictions. By disallowing number restrictions and nominals, we obtain $\mathcal{S H \mathcal { I }}$. Finally, $\mathcal{S H O I} \sqcap$ minus nominals and inverse roles, results in the DL $\mathcal{S H} \mathcal{H} \mathcal{Q}^{\sqcap}$.

A general concept inclusion (GCI) is an expression $C \sqsubseteq D$, where both $C$ and $D$ are concepts. A finite set of GCIs is called a TBox. An interpretation $\mathcal{I}$ satisfies a $G C I C \sqsubseteq D$ if $C^{\mathcal{I}} \subseteq D^{\mathcal{I}}$, and a TBox $\mathcal{T}$ if it satisfies every GCI in $\mathcal{T}$.

An (ABox) assertion is an expression of the form $C(a), r(a, b)$, where $C$ is a concept, $r$ a role, and $a, b \in N_{I}$. An $A B o x$ is a finite set of assertions. We use $N_{I}(\mathcal{A})$ to denote the set of individual names occurring in $\mathcal{A}$. An interpretation $\mathcal{I}$ satisfies an assertion $C(a)$ if $a^{\mathcal{I}} \in C^{\mathcal{I}}, r(a, b)$ if $\left\langle a^{\mathcal{I}}, b^{\mathcal{I}}\right\rangle \in r^{\mathcal{I}}$. An interpretation $\mathcal{I}$ satisfies an $A B o x \mathcal{A}$ if it satisfies each assertion in $\mathcal{A}$, denoted as $\mathcal{I} \models \mathcal{A}$.

A knowledge base $(\mathrm{KB})$ is a triple $(\mathcal{R}, \mathcal{T}, \mathcal{A})$ with $\mathcal{R}$ a role hierarchy, $\mathcal{T}$ a TBox, and $\mathcal{A}$ an ABox. Let $\mathcal{K}=(\mathcal{R}, \mathcal{T}, \mathcal{A})$ be a knowledge base and $\mathcal{I}=\left(\Delta^{\mathcal{I}},{ }^{\mathcal{I}}\right)$ an interpretation. We say that $\mathcal{I}$ satisfies $\mathcal{K}$ if $\mathcal{I}$ satisfies $\mathcal{R}, \mathcal{I}$, and $\mathcal{A}$. In this case, we say that $\mathcal{I}$ is a model of $\mathcal{K}$ and write $\mathcal{I} \models \mathcal{K}$. We say that $\mathcal{K}$ is satisfiable if $\mathcal{K}$ has a model. A concept $D$ subsumes a concept $C$ w.r.t. $\mathcal{K}$ if $C^{\mathcal{I}} \subseteq D^{\mathcal{I}}$ for every model $\mathcal{I}$ of $\mathcal{K}$. A concept $C$ is satisfiable w.r.t. $\mathcal{K}$ if there is a model $\mathcal{I}$ of $\mathcal{K}$ such that $C^{\mathcal{I}} \neq \emptyset$. It should be noted that the standard reasoning tasks such as knowledge base satisfiability, concept subsumption, or concept satisfiability are mutually reducible in polynomial time.

\section{$3 \mathcal{S H} \mathcal{Q}^{\square}$ is ExpTime-complete}

In this section, we show that adding role conjunctions to the $\mathrm{DL} \mathcal{S H \mathcal { Q }}$ does not increase the computational complexity of the standard reasoning tasks. For this purpose, we devise a polynomial translation of a given $\mathcal{S H} \mathcal{Q}^{\sqcap}$ knowledge base to an equisatisfiable $\mathcal{A L C H} \mathcal{Q}^{\sqcap}$ knowledge base (i.e., $\mathcal{S H} \mathcal{Q}^{\sqcap}$ minus role transitivity) for which the standard reasoning tasks are ExpTime-complete $[6,3,7]$.

Let $\mathcal{K}=(\mathcal{R}, \mathcal{T}, \mathcal{A})$ be an $\mathcal{S H} \mathcal{Q}^{\sqcap}$ knowledge base. We say that $\mathcal{K}$ is simplified if $\mathcal{T}$ contains only axioms of the form:

$$
A \sqsubseteq \forall \rho . B|A \sqsubseteq \exists \rho . B| A \sqsubseteq \bowtie n \delta . B \mid \bigcap A_{i} \sqsubseteq \bigsqcup B_{j},
$$

where $A_{(i)}$ and $B_{(j)}$ are atomic concepts, $\rho(\delta)$ is a (simple) conjunction of roles, and $\bowtie$ stands for $\leqslant$ or $\geqslant$. Furthermore, concept assertions in $\mathcal{A}$ are limited to the 
form $A(a)$ for $A$ a concept name. Every $\mathcal{S H} \mathcal{Q}^{\sqcap}$ knowledge base, which is not in this form, can be transformed in polynomial time into the desired form by using the standard structural transformation, which iteratively introduces definitions for compound sub-concepts and sub-roles (see, e.g., [8]).

It is well known that transitivity can be eliminated from $\mathcal{S H I} \mathcal{Q}$ and $\mathcal{S H O I Q}$ knowledge bases by using auxiliary axioms that propagate the concepts over transitive roles $[8,9]$. The transitivity elimination has been extended to $\mathcal{S H \mathcal { L }}{ }^{\sqcap}$ [3], however it becomes exponential in the worst case, since one has to introduce new axioms for (possibly exponentially many) conjunctions of transitive roles. The procedure is, however, polynomial if either the number of transitive roles in role inclusions or the length of role conjunctions is bounded. Below we describe a polynomial elimination of transitivity when there are no role inverses.

Let $\mathcal{K}=(\mathcal{R}, \mathcal{T}, \mathcal{A})$ be a simplified $\mathcal{S H} \mathcal{Q}^{\sqcap}$ knowledge base. We construct an $\mathcal{A L C H} \mathcal{Q}^{\square}$ knowledge base $\mathcal{K}^{\prime}=\left(\mathcal{R}^{\prime}, \mathcal{T}^{\prime}, \mathcal{A}^{\prime}\right)$ from $\mathcal{K}$ as follows. The signature of $\mathcal{K}^{\prime}$ is defined by $N_{I}\left(\mathcal{K}^{\prime}\right):=N_{I}(\mathcal{K}), N_{R}\left(\mathcal{K}^{\prime}\right):=N_{R}(\mathcal{K}), N_{t R}\left(\mathcal{K}^{\prime}\right):=\emptyset, N_{C}\left(\mathcal{K}^{\prime}\right):=$ $N_{C}(\mathcal{K}) \cup\left\{A_{a}, A_{a}^{r} \mid A \in N_{C}(\mathcal{K}), a \in N_{I}(\mathcal{K}), r \in N_{R}(\mathcal{K})\right\}$. Recall, that w.l.o.g., $N_{I}(\mathcal{K})$ is non-empty, therefore there exists at least one $A_{a}$ for every $A \in N_{C}(\mathcal{K})$. We set $\mathcal{R}^{\prime}:=\mathcal{R}, \mathcal{A}^{\prime}:=\mathcal{A}$, and $\mathcal{T}^{\prime}$ as an extension of $\mathcal{T}$ with the following axioms:

$$
\begin{array}{rr}
A \sqsubseteq \bigsqcup_{a \in N_{I}(\mathcal{A})} A_{a} & A \in N_{C}(\mathcal{K}) \\
A_{a} \sqsubseteq \forall r . A_{a}^{r} & A \in N_{C}(\mathcal{K}), a \in N_{I}(\mathcal{A}), r \in N_{R}(\mathcal{K}) \\
A_{a}^{t} \sqsubseteq \forall t . A_{a}^{t} & A \in N_{C}(\mathcal{K}), a \in N_{I}(\mathcal{A}), t \in N_{t R}(\mathcal{K}) \\
A_{a}^{t} \sqsubseteq A_{a}^{r} & A \in N_{C}(\mathcal{K}), a \in N_{I}(\mathcal{A}), t \in N_{t R}(\mathcal{K}), r \in N_{R}(\mathcal{K}), t \sqsubseteq \mathcal{R} r \\
A_{a}^{r_{1}} \sqcap \cdots \sqcap A_{a}^{r_{n}} \sqsubseteq B \quad a \in N_{I}(\mathcal{A}),(A \sqsubseteq \forall \rho . B) \in \mathcal{T}, \rho=r_{1} \sqcap \cdots \sqcap r_{n}
\end{array}
$$

Theorem 1. Let $\mathcal{K}=(\mathcal{R}, \mathcal{T}, \mathcal{A})$ be a simplified $\mathcal{S H} \mathcal{Q}^{\square}$ knowledge base and $\mathcal{K}^{\prime}=$ $\left(\mathcal{R}^{\prime}, \mathcal{T}^{\prime}, \mathcal{A}^{\prime}\right)$ an $\mathcal{A L C H} \mathcal{Q}^{\square}$ knowledge base obtained from $\mathcal{K}$ as described above. Then $(i) \mathcal{K}^{\prime}$ is obtained from $\mathcal{K}$ in polynomial time and (ii) $\mathcal{K}$ is satisfiable iff $\mathcal{K}^{\prime}$ is satisfiable.

Proof (Sketch). Claim (i) is straightforward. We sketch the proof for Claim (ii).

For the "if" direction of (ii), one can show that every model $\mathcal{J}$ of $\mathcal{K}^{\prime}$ can be extended to a model $\mathcal{I}$ of $\mathcal{K}$ by interpreting non-simple roles $r \in N_{R}$ as $r^{\mathcal{J}} \cup \bigcup_{t \sqsubseteq \mathcal{R} r} r, t \in N_{t R}\left(t^{\mathcal{J}}\right)^{+}$and leaving the interpretation of the other symbols unchanged. All axioms that do not have negative occurrences of non-simple roles remain satisfied in $\mathcal{I}$. Among the remaining axioms are RIAs $r \sqsubseteq s$ and axioms of the form $A \sqsubseteq \forall \rho$.B. RIAs $r \sqsubseteq s$ are satisfied by definition of $\mathcal{I}$, and axioms of the form $A \sqsubseteq \forall \rho . B$ are satisfied due to axioms (1)-(5).

For the "only if" direction of (ii) we use the fact that every satisfiable $\mathcal{S H} \mathcal{Q}^{\sqcap}$ knowledge base $\mathcal{K}$ has a forest-shaped model $\mathcal{I}$, where the ABox individuals form the roots of the trees and relations can only be between the individuals or within the trees. The model $\mathcal{I}$ can be then extended to a model $\mathcal{J}$ of the axioms $(1)-(5)$ by interpreting $A_{a}$ as the restriction of $A$ to the elements of the tree growing from $a$, and $A_{a}^{r}$ as the minimal sets satisfying axioms (2)-(4). For proving that $\mathcal{J}$ satisfies all axioms of the form (5), we use a property that if two elements of a tree have a common descendant, then one is a descendant of the other. 
Corollary 1. The problem of concept satisfiability in the $D L \mathcal{S H} \mathcal{Q}^{\sqcap}$ is ExpTimecomplete (and so are all the standard reasoning problems).

\section{$4 \mathcal{S H} \mathcal{H} \mathcal{I}^{\sqcap}$ is 2ExpTime-complete}

In this section, we show that extending $\mathcal{S H \mathcal { I }}$ with role conjunctions causes an exponential blow-up in the computational complexity of the standard reasoning tasks. We show this by a reduction from the word problem of an exponential space alternating Turing machine.

An alternating Turning machine (ATM) is a tuple $M=\left(\Gamma, \Sigma, Q, q_{0}, \delta_{1}, \delta_{2}\right)$, where $\Gamma$ is a finite working alphabet containing a blank symbol $\square, \Sigma \subseteq \Gamma \backslash\{\square\}$ is the input alphabet; $Q=Q_{\exists} \uplus Q_{\forall} \uplus\left\{q_{a}\right\} \uplus\left\{q_{r}\right\}$ is a finite set of states partitioned into existential states $Q_{\exists}$, universal states $Q_{\forall}$, an accepting state $q_{a}$, and a rejecting state $q_{r} ; q_{0} \in Q_{\exists}$ is the starting state, and $\delta_{1}, \delta_{2}:\left(Q_{\exists} \cup Q_{\forall}\right) \times \Gamma \rightarrow$ $Q \times \Gamma \times\{L, R\}$ are transition functions. A configuration of $M$ is a word $c=$ $w_{1} q w_{2}$ where $w_{1}, w_{2} \in \Gamma^{*}$ and $q \in Q$. An initial configuration is $c^{0}=q_{0} w_{0}$ where $w_{0} \in \Sigma^{*}$. The size $|c|$ of a configuration $c$ is the number of symbols in $c$. The successor configurations $\delta_{1}(c)$ and $\delta_{2}(c)$ of a configuration $c=w_{1} q w_{2}$ with $q \neq q_{a}, q_{r}$ over the transition functions $\delta_{1}$ and $\delta_{2}$ are defined as for deterministic Turing machines (see, e.g., [10]). The sets $\mathrm{C}_{\mathrm{acc}}(M)$ of accepting configurations and $\mathrm{C}_{\text {rej }}(M)$ of rejecting configurations of $M$ are the smallest sets such that (i) $c=w_{1} q w_{2} \in \mathrm{C}_{\mathrm{acc}}(M)$ if either $q=q_{a}$, or $q \in Q_{\forall}$ and $\delta_{1}(c), \delta_{2}(c) \in \mathrm{C}_{\mathrm{acc}}(M)$, or $q \in Q_{\exists}$ and $\delta_{1}(c) \in \mathrm{C}_{\mathrm{acc}}(M)$ or $\delta_{2}(c) \in \mathrm{C}_{\mathrm{acc}}(M)$, and (ii) $c=w_{1} q w_{2} \in$ $\mathrm{C}_{\text {rej }}(M)$ if either $q=q_{r}$, or $q \in Q_{\exists}$ and $\delta_{1}(c), \delta_{2}(c) \in \mathrm{C}_{\text {rej }}(M)$, or $q \in Q_{\forall}$ and $\delta_{1}(c) \in \mathrm{C}_{\text {rej }}(M)$ or $\delta_{2}(c) \in \mathrm{C}_{\text {rej }}(M)$. The set of reachable configurations from an initial configuration $c^{0}$ in $M$ is the smallest set $M\left(c^{0}\right)$ such that $c^{0} \in$ $M\left(c^{0}\right)$ and $\delta_{1}(c), \delta_{2}(c) \in M\left(c^{0}\right)$ for every $c \in M\left(c^{0}\right)$. A word problem for an ATM $M$ is to decide given an initial configuration $c^{0}$ whether $c^{0} \in \mathrm{C}_{\text {acc }}(M)$. $M$ is $g(n)$ space bounded if for every initial configuration $c^{0}$ we have: (i) $c^{0} \in$ $\mathrm{C}_{\text {acc }}(M) \cup \mathrm{C}_{\text {rej }}(M)$, and (ii) $|c| \leq g\left(\left|c^{0}\right|\right)$ for every $c \in M\left(c^{0}\right)$. A classical result AExpSpace $=2$ ExpTime [11] implies that there exists a $2^{n}$ space bounded ATM $M$ for which the following decision problem is 2ExpTime-complete: given an initial configuration $c^{0}$ decide whether $c^{0} \in \mathrm{C}_{\mathrm{acc}}(M)$.

We encode a computation of the ATM $M$ in a binary tree (see Figure 1) whereby the configurations of $M$ are encoded on exponentially long chains that grow from the nodes of the tree - the $i^{\text {th }}$ element of a chain represents the $i^{\text {th }}$ element of the configuration. In our construction, we distinguish odd and even configurations in the computation using concept names Odd and Even. Every odd configuration has two even successor configurations reachable by roles $r_{e}^{1}$ and $r_{e}^{2}$ respectively; likewise, every even configuration has two odd successor configurations reachable by inverses of $r_{o}^{1}$ and $r_{o}^{2}$. We further alternate between the concepts $P_{0}, P_{1}$, and $P_{2}$ within the levels of the binary tree. This allows us to distinguish the predecessor and the successor configuration represented by the exponentially long chains. We enforce these chains (see Figure 2) by using the well know "integer counting" technique [12]. A counter $c^{\mathcal{I}}(x)$ is an integer 

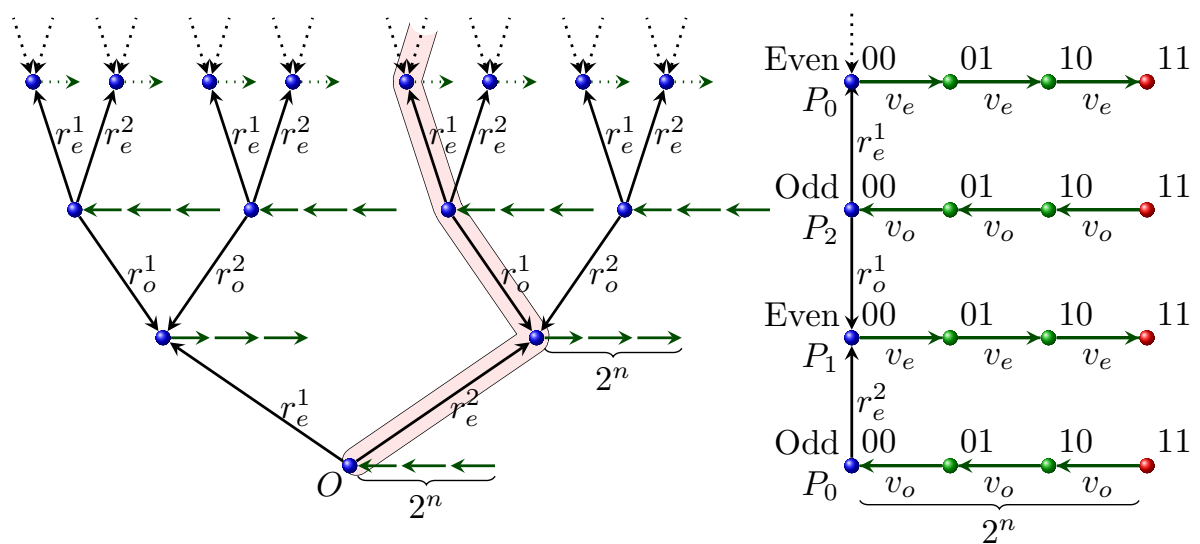

Fig. 1. The alternating binary tree structure for simulating a computation of the ATM (left) and a detailed picture for the highlighted path (right)

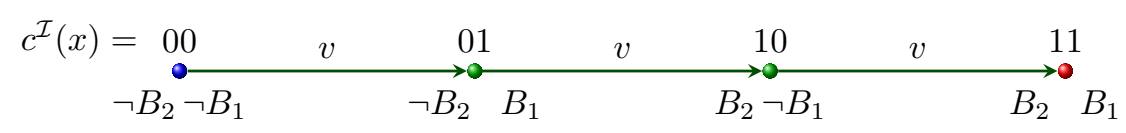

Fig. 2. Expressing exponentially long chains using a counter and binary encoding

between 0 and $2^{n}-1$ that is assigned to an element $x$ of the interpretation $\mathcal{I}$ using $n$ atomic concepts $B_{1}, \ldots, B_{n}$ such that the $i^{\text {th }}$ bit of $c^{\mathcal{I}}(x)$ is equal to 1 iff $x \in B_{i}{ }^{\mathcal{I}}$. We first define the concept $Z$ that can be used to initialize the counter to zero, and the concept $E$ to detect whether the counter has reached the final value $2^{n}-1$ and, thus, the end of the chain is reached:

$$
Z \equiv \neg B_{1} \sqcap \ldots \sqcap \neg B_{n} \quad E \equiv B_{1} \sqcap \ldots \sqcap B_{n}
$$

Every element that is not the end of the chain has a $v$-successor:

$$
\neg E \sqsubseteq \exists v . \top
$$

The lowest bit of the counter is always flipped over $v$, while any other bit of the counter is flipped over $v$ if and only if the previous bit is flipped from 1 to 0 :

$$
\begin{aligned}
\top & \equiv\left(B_{1} \sqcap \forall v \cdot \neg B_{1}\right) \sqcup\left(\neg B_{1} \sqcap \forall v \cdot B_{1}\right) \\
B_{k-1} \sqcap \forall v \cdot \neg B_{k-1} & \equiv\left(B_{k} \sqcap \forall v \cdot \neg B_{k}\right) \sqcup\left(\neg B_{k} \sqcap \forall v \cdot B_{k}\right) \quad 1<k \leq n
\end{aligned}
$$

For convenience, let us denote by $j[i]_{2}$ the $i^{\text {th }}$ bit of $j$ in binary coding (the lowest bit of $j$ is $j[1]_{2}$ ).

The tree-like structure in Figure 1 is induced by the following formulas. First, we initialize the origin $O$ of the tree by saying that it belongs to an odd row labeled with $P_{0}$ and, with the concept $Z$, we initialize an exponential chain:

$$
O \sqsubseteq \text { Odd } \sqcap P_{0} \sqcap Z
$$


Every initial element of an exponential chain has two successors alternating between odd and even values:

$$
\begin{gathered}
Z \sqcap \text { Odd } \sqsubseteq \exists r_{e}^{1} \text {. Even } \sqcap \exists r_{e}^{2} \text {.Even } \\
Z \sqcap \text { Even } \sqsubseteq \exists r_{o}^{1-} \text {.Odd } \sqcap \exists r_{o}^{2-} \text {.Odd }
\end{gathered}
$$

For convenience, we introduce super-roles $r^{1}, r^{2}$ and $r$ of the created roles to keep track of the relations between the nodes and their successors:

$$
r_{e}^{1} \sqsubseteq r^{1} \quad r_{o}^{1} \sqsubseteq r^{1^{-}} \quad r_{e}^{2} \sqsubseteq r^{2} \quad r_{o}^{2} \sqsubseteq r^{2^{-}} \quad r^{1} \sqsubseteq r \quad r^{2} \sqsubseteq r
$$

The new roles are used to initialize the value $Z$ for the successors and increment $P_{j}$ over $r$ modulo 3 (we denote $j+1 \bmod 3$ as $[j+1]_{3}$ ):

$$
Z \sqsubseteq \forall r . Z \quad P_{j} \sqsubseteq \forall r . P_{[j+1]_{3}} \quad 0 \leq j \leq 2
$$

In order to have the roles on the exponential chain correspond to the odd and even rows, we replace axiom (7) with the following axioms:

$$
\begin{aligned}
\neg E \sqcap \text { Even } & \sqsubseteq \exists v_{e} \cdot \top & \neg E \sqcap \text { Odd } & \sqsubseteq \exists v_{o}^{-} \cdot \top \\
v_{o} & \sqsubseteq v^{-} & v_{e} & \sqsubseteq v \\
\text { Odd } & \sqsubseteq \forall v \text {.Odd } & \text { Even } & \sqsubseteq \forall v . \text { Even }
\end{aligned}
$$

The values of $P_{j}$ are copied across the elements of the same row:

$$
P_{j} \sqsubseteq \forall v \cdot P_{j} \quad \neg P_{j} \sqsubseteq \forall v \cdot \neg P_{j} \quad 0 \leq j \leq 2
$$

If we take a look at Figure 1, we notice that the roles $r_{o}^{i}, r_{e}^{i}, v_{o}$ and $v_{e}$ are directed in such a way that, from every element of an exponential chain, only elements of the neighboring chains are reachable by a sequence of roles. In other words, if we introduce a common transitive super-role $t$ of these roles, then every element of the chain will be connected via $t$ to exactly all elements of the parent chain and all elements of the successor chains. Unfortunately, this is not sufficient to simulate a computation of the Turing machine, as we need to connect exactly the corresponding elements of a chain and its two successor chains to compute the successor configurations. In order to achieve this goal, we will add auxiliary chains to the exponential chain that, using transitive superroles and role conjunctions, will allow us to restrict the reachability relation only to the corresponding elements.

The detailed construction for the side chains of two successive configurations is shown in Figure 3. Every element of the exponential $v$-chain has $n$ additional "side" successors reachable by roles $h_{k e}^{j}$ and $h_{k o}^{j}$ with $j \in\{0,1\}$ and $1 \leq k \leq n$. Intuitively, $k$ corresponds to the counting concepts and $j$ to the counter value. We will also count the level in the $h$-chains using concepts $H_{k}, 0 \leq k \leq n$-all elements of the $v$-chain belong to $H_{0}$, and every $h$-successor of an element in 


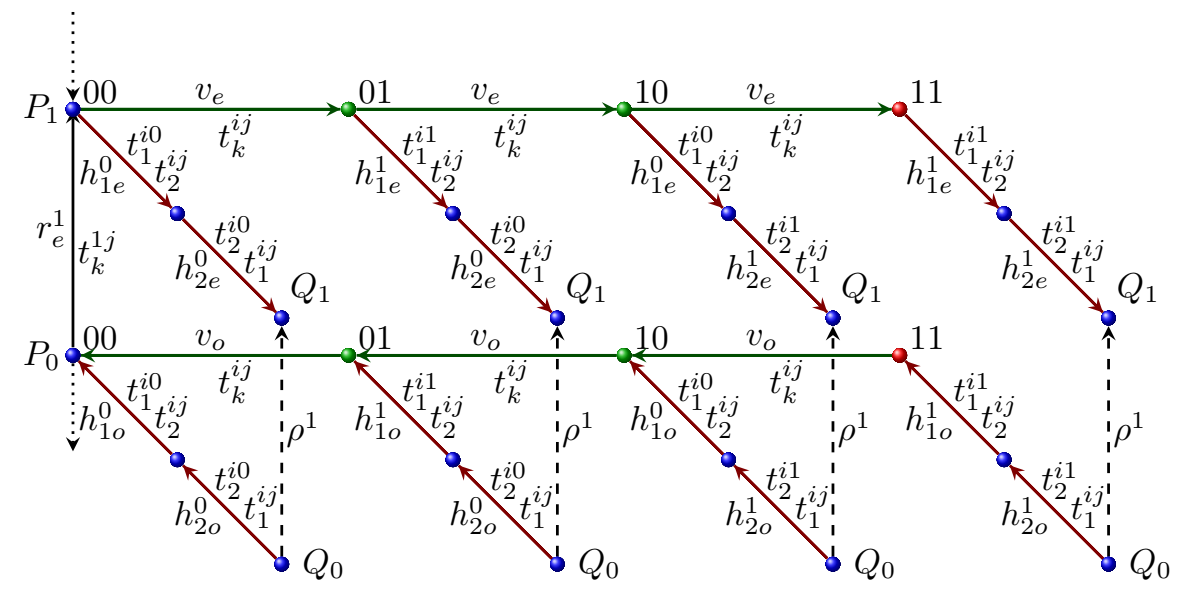

Fig. 3. A zoom-in and extension of Figure 1, which illustrates the use of the auxiliary side chains to connect the elements of the exponentially long chains with the corresponding elements in the successor chains

$H_{k-1}$ belongs to $H_{k}$. The following axioms initialize the side chains according to this description:

$$
\begin{array}{rlrl}
O & \sqsubseteq H_{0} \quad H_{0} \sqsubseteq \forall r \cdot H_{0} \quad H_{0} \sqsubseteq \forall v \cdot H_{0} & \\
H_{k-1} \sqcap \neg B_{k} & \sqsubseteq\left(\neg \text { Even } \sqcup \exists h_{k e}^{0} \cdot H_{k}\right) \sqcap\left(\neg \text { Odd } \sqcup \exists h_{k o}^{0}{ }^{-} \cdot H_{k}\right) & 1 \leq k \leq n \\
H_{k-1} \sqcap B_{k} & \sqsubseteq\left(\neg \text { Even } \sqcup \exists h_{k e}^{1} \cdot H_{k}\right) \sqcap\left(\neg \text { Odd } \sqcup \exists h_{k o}^{1}{ }^{-} \cdot H_{k}\right) & 1 \leq k \leq n \\
h_{k e}^{j} & \sqsubseteq h \quad \quad h_{k o}^{j} \sqsubseteq h^{-} \quad j \in\{0,1\}, 1 \leq k \leq n \\
\text { Even } & \sqsubseteq \forall h \text {.Even } \quad \text { Odd } \sqsubseteq \forall h \text {.Odd } &
\end{array}
$$

We use these roles to express that the elements within an $h$-chain have the same values for $B_{k}$ and $P_{j}$ :

$$
\begin{array}{lll}
B_{k} \sqsubseteq \forall h . B_{k} & \neg B_{k} \sqsubseteq \forall h . \neg B_{k} & 0 \leq k \leq n \\
P_{j} \sqsubseteq \forall h . P_{j} & \neg P_{j} \sqsubseteq \forall h . \neg P_{j} & 0 \leq j \leq 2
\end{array}
$$

For the final elements of the $h$-chains, we introduce the special concepts $Q_{i}$ that correlate with the concepts $P_{j}$ :

$$
H_{n} \sqsubseteq\left(P_{j} \sqcap Q_{j}\right) \sqcup\left(\neg P_{j} \sqcap \neg Q_{j}\right) \quad 0 \leq j \leq 2
$$

These concepts will be used to connect the last elements of the $h$-chains with the corresponding elements in the chains for the two successor configurations using role conjunctions $\rho^{1}$ and $\rho^{2}$ introduced later on (see Figure 3). In order to connect these elements, we introduce transitive super-roles $t_{k}^{i j}$ with $i \in\{1,2\}$, 
$j \in\{0,1\}$, and $1 \leq k \leq n$ :

$$
\begin{array}{rlrl}
r_{o}^{i} & \sqsubseteq t_{k}^{i j} & r_{e}^{i} \sqsubseteq t_{k}^{i j} & \\
v_{o} \sqsubseteq t_{k}^{i j} & v_{e} \sqsubseteq t_{k}^{i j} \\
h_{k o}^{j} \sqsubseteq t_{k}^{i j} & h_{k e}^{j} \sqsubseteq t_{k}^{i j} & \\
h_{k o}^{j} & \sqsubseteq t_{k^{\prime}}^{i j^{\prime}} & h_{k e}^{j} \sqsubseteq t_{k^{\prime}}^{i j^{\prime}} & j^{\prime} \in\{0,1\}, 1 \leq k^{\prime} \leq n, k^{\prime} \neq k
\end{array}
$$

Intuitively, the index $i$ in $t_{k}^{i j}$ is inherited from the roles $r_{o}^{i}$ and $r_{e}^{i}(27)$-all role implications hold for both values of $i$. Likewise, the index $j$ is inherited from $h_{k o}^{j}$ and $h_{k e}^{j}$, but only when the values of the index $k$ match (29) - otherwise the role implications hold for both values of $j(30)$. Roles $v_{o}$ and $v_{e}$ do not filter any indexes and imply all roles $t_{k}^{i j}(28)$. Axioms (27)-(30) make sure that the first and the last elements of every $h$-chain are connected with $t_{k}^{i 0}\left(t_{k}^{i 1}\right)$ iff the $k^{\text {th }}$ bit of the counter is 0 (1). Thus, only the corresponding last elements of the $h$-chains in the successor configurations are connected with $t_{k}^{i j}$ for all $k$ with $1 \leq k \leq n$ and some $i$ and $j$, because they have the same values for the counter. To make use of this property we introduce roles $s_{k}^{i}$ that are obtained from $t_{k}^{i j}$ by abstracting from $j$ and forgetting the direction:

$$
t_{k}^{i j} \sqsubseteq s_{k}^{i} \quad t_{k}^{i j^{-}} \sqsubseteq s_{k}^{i} \quad i \in\{1,2\}, j \in\{0,1\}, 1 \leq k \leq n
$$

Now define the role conjunctions $\rho^{1}=s_{1}^{1} \sqcap \cdots \sqcap s_{n}^{1}$ and $\rho^{2}=s_{1}^{2} \sqcap \cdots \sqcap s_{n}^{2}$ that connect the last elements of the $h$-chains iff they are the corresponding elements for the $r^{1}$ and $r^{2}$ successors in our binary tree on Figure 1. Note that $\rho^{1}$ and $\rho^{2}$ are not simple.

We now specify how the created tree structure relates to an alternating Turing machine. Let $c^{0}$ be an initial configuration of an ATM $M=\left(\Gamma, \Sigma, Q, q_{0}, \delta_{1}, \delta_{2}\right)$ and $n=\left|c^{0}\right|$ (w.l.o.g., we assume that $n>2$ ). In order to decide whether $c^{0} \in \mathrm{C}_{\text {acc }}(M)$, we try to build all the required accepting successor configurations of $c^{0}$ for $\mathrm{M}$. We encode the configurations of $M$ on the $2^{n}$-long $v$-chains. A chain corresponding to a configuration $c$ is connected via the roles $r^{1}$ and $r^{2}$ to two chains that correspond to $\delta_{1}(c)$ and $\delta_{2}(c)$ respectively. We use an atomic concept $A_{a}$ for every symbol $a$ that can occur in configurations and we make sure that all elements of the same $h$-chain are assigned to the same symbol:

$$
A_{a} \sqsubseteq \forall h . A_{a} \quad \neg A_{a} \sqsubseteq \forall h . \neg A_{a}
$$

It is a well-known property of the transition functions of Turing machines that the symbols $c_{i}^{1}$ and $c_{i}^{2}$ at the position $i$ of $\delta_{1}(c)$ and $\delta_{2}(c)$ are uniquely determined by the symbols $c_{i-1}, c_{i}, c_{i+1}$, and $c_{i+2}$ of $c$ at the positions $i-1, i, i+1$, and $i+2 .{ }^{1}$ We assume that this correspondence is given by the (partial) functions $\lambda_{1}$ and $\lambda_{2}$ such that $\lambda_{1}\left(c_{i-1}, c_{i}, c_{i+1}, c_{i+2}\right)=c_{i}^{1}$ and $\lambda_{2}\left(c_{i-1}, c_{i}, c_{i+1}, c_{i+2}\right)=c_{i}^{2}$. We use this property in our encoding as follows: for every quadruple of symbols

\footnotetext{
${ }^{1}$ If any of the indexes $i-1, i+1$, or $i+2$ are out of range for the configuration $c$, we assume that the corresponding symbols $c_{i-1}, c_{i+1}$, and $c_{i+2}$ are the blank symbol $\square$.
} 
$a_{1}, a_{2}, a_{3}, a_{4} \in Q \cup \Gamma$, we introduce a concept name $S_{a_{1} a_{2} a_{3} a_{4}}$ which expresses that the current element of the $v$-chain is assigned with the symbol $a_{2}$, its $v$ predecessor with $a_{1}$ and its next two $v$-successors with respectively $a_{3}$ and $a_{4}$ $\left(a_{1}, a_{3}\right.$, and $a_{4}$ are $\square$ if there are no such elements):

$$
\begin{array}{rrr}
Z \sqcap A_{a_{2}} \sqcap \exists v \cdot\left(A_{a_{3}} \sqcap \exists v \cdot A_{a_{4}}\right) & \sqsubseteq S_{\varpi a_{2} a_{3} a_{4}} & a_{2}, a_{3}, a_{4} \in Q \cup \Gamma \\
A_{a_{1}} \sqcap \exists v \cdot\left(A_{a_{2}} \sqcap \exists v \cdot\left(A_{a_{3}} \sqcap \exists v \cdot A_{a_{4}}\right)\right) & \sqsubseteq \forall v \cdot S_{a_{1} a_{2} a_{3} a_{4}} & a_{1}, a_{2}, a_{3}, a_{4} \in Q \cup \Gamma \\
A_{a_{1}} \sqcap \exists v \cdot\left(A_{a_{2}} \sqcap \exists v \cdot\left(A_{a_{3}} \sqcap E\right)\right) & \sqsubseteq \forall v \cdot S_{a_{1} a_{2} a_{3}} \boxminus & a_{1}, a_{2}, a_{3} \in Q \cup \Gamma \\
A_{a_{1}} \sqcap \exists v \cdot\left(A_{a_{2}} \sqcap E\right) & \sqsubseteq \forall v \cdot S_{a_{1} a_{2}} \boxminus \square & a_{1}, a_{2} \in Q \cup \Gamma
\end{array}
$$

Furthermore, all elements of the same $h$-chain have the same values of $S_{a_{1} a_{2} a_{3} a_{4}}$ :

$$
S_{a_{1} a_{2} a_{3} a_{4}} \sqsubseteq \forall h . S_{a_{1} a_{2} a_{3} a_{4}} \quad \neg S_{a_{1} a_{2} a_{3} a_{4}} \sqsubseteq \forall h . \neg S_{a_{1} a_{2} a_{3} a_{4}}
$$

Finally, the properties of the transition functions are expressed using the following axioms, where, as previously defined $\rho^{1}=s_{1}^{1} \sqcap \cdots \sqcap s_{n}^{1}$ and $\rho^{2}=s_{1}^{2} \sqcap \cdots \sqcap s_{n}^{2}$ :

$$
\begin{array}{ll}
S_{a_{1} a_{2} a_{3} a_{4}} \sqcap Q_{j} \sqsubseteq \forall \rho^{1} \cdot\left[\neg Q_{[j+1]_{3}} \sqcup A_{\lambda_{1}\left(a_{1}, a_{2}, a_{3}, a_{4}\right)}\right] & 0 \leq i \leq 2 \\
S_{a_{1} a_{2} a_{3} a_{4}} \sqcap Q_{j} \sqsubseteq \forall \rho^{2} \cdot\left[\neg Q_{[j+1]_{3}} \sqcup A_{\lambda_{2}\left(a_{1}, a_{2}, a_{3}, a_{4}\right)}\right] & 0 \leq i \leq 2
\end{array}
$$

Intuitively, these axioms say that whenever $S_{a_{1} a_{2} a_{3} a_{4}}$ holds at the end of an $h$ chain where $Q_{j}$ holds, then $A_{\lambda_{1}\left(a_{1}, a_{2}, a_{3}, a_{4}\right)}$ should hold for every $\rho^{1}\left(\rho^{2}\right)$ successor for which $Q_{[j+1]_{3}}$ holds. As noted before, only the corresponding last elements of the $h$-chains can be connected by $\rho^{1}$ and $\rho^{2}$. The concepts $Q_{j}$ and $Q_{[j+1]_{3}}$ restrict the attention to the last elements of the $h$-chains and make sure that the information is propagated to the successor configuration and not to the predecessor configuration.

We now make sure that the elements in the root chain of our tree correspond to the initial configuration $c^{0}$ :

$$
\begin{aligned}
O & \sqsubseteq A_{c_{1}^{0}} \sqcap \forall v \cdot\left(A_{c_{2}^{0}} \sqcap \cdots \forall v \cdot\left(A_{c_{n}^{0}} \sqcap \forall v \cdot O_{\varpi}\right) \cdots\right) \\
O_{\square} & \sqsubseteq A_{\varpi} \sqcap \forall v \cdot O_{\square}
\end{aligned}
$$

In order to distinguish between the configurations with existential and universal states, we introduce two concepts $S_{\forall}$ and $S_{\exists}$, which are implied by the corresponding states and propagated to the first elements of the configuration:

$$
\begin{aligned}
A_{q} & \sqsubseteq S_{\exists} & q \in Q_{\exists} & A_{q} \sqsubseteq S_{\forall} \\
\exists v . S_{\exists} & \sqsubseteq S_{\exists} & & \exists v . S_{\forall} \sqsubseteq S_{\forall}
\end{aligned}
$$

Now instead of always creating two successor configurations, we create only configurations that are required for acceptance. Thus, we replace axioms (11) and (12) with the axioms (44)-(46) below:

$$
\begin{array}{cc}
Z \sqcap \text { Odd } \sqcap S_{\forall} \sqsubseteq \exists r_{e}^{1} \cdot \top \sqcap \exists r_{e}^{2} \cdot \top & Z \sqcap \text { Even } \sqcap S_{\forall} \sqsubseteq \exists r_{o}^{1-} \cdot \top \sqcap \exists r_{o}^{2-} \cdot \top \\
Z \sqcap \text { Odd } \sqcap S_{\exists} \sqsubseteq \exists r_{e}^{1} \cdot \top \sqcup \exists r_{e}^{2} \cdot \top & Z \sqcap \text { Even } \sqcap S_{\exists} \sqsubseteq \exists r_{o}^{1-} \cdot \top \sqcup \exists r_{o}^{2-} \cdot \top \\
\text { Odd } \sqsubseteq \forall r . \text { Even } & \text { Even } \sqsubseteq \forall r . \text { Odd }
\end{array}
$$


Finally we forbid configurations with rejecting states in our model:

$$
A_{q_{r}} \sqsubseteq \perp
$$

To summarize, our construction proves the following theorem:

Theorem 2. Let $c^{0}$ be an initial configuration for the ATM $M$ and $\mathcal{K}$ a knowledge base consisting of the axioms (6)-(10) and (13)-(47). Then $c^{0} \in \mathrm{C}_{\mathrm{acc}}(M)$ if and only if $O$ is (finitely) satisfiable in $\mathcal{K}$.

When analyzing the number of introduced axioms and their size, we see that their number is polynomial in $n$ and their size is linear in $n$, where $n$ is the size of the initial configuration. Hence, we get the following result.

Corollary 2. The problem of (finite) concept satisfiability in the $D L \mathcal{S H} \mathcal{I}^{\sqcap}$ is 2ExpTime-hard (and so are all the standard reasoning problems).

The corresponding upper bound from [3] gives us the following result.

Corollary 3. The problem of concept satisfiability in $\mathcal{S H \mathcal { I }}{ }^{\square}$ and $\mathcal{S H I} \mathcal{Q}^{\square}$ is 2ExpTime-complete (and so are all the standard reasoning problems).

Since the problem of KB satiafiability in $\mathcal{S H} \mathcal{H}^{\sqcap}$ can be polynomially reduced to non-entailment of a union of conjunctive queries with at most two variables [5], we also get the following result.

Corollary 4. The problem of entailment for unions of conjunctive queries in $\mathcal{S H I}$ is 2ExpTime-complete already for queries with at most two variables.

\section{$5 \mathcal{S H O \mathcal { I F }} \mathcal{F}^{\sqcap}$ is N2ExpTime-hard}

For proving the lower bound of reasoning in $\mathcal{S H \mathcal { H } \mathcal { I }} \sqcap$, we use a reduction from the double exponential domino tiling problem. We demonstrate how, by using $\mathcal{S H O \mathcal { I }}{ }^{\square}$ formulas, one can encode a $2^{2^{n}} \times 2^{2^{n}}$ grid-like structure illustrated in Figure 4. As in our tree-like structure in Figure 1 we will use four roles $r_{o}^{1}$, $r_{2}^{1}, r_{o}^{2}$, and $r_{e}^{2}$ with alternating directions to create the grid. Roles $r_{o}^{1}$ and $r_{e}^{1}$ induce horizontal edges and roles $r_{o}^{2}$ and $r_{e}^{2}$ induce vertical edges. The nodes of the grid are also partitioned on even and odd in a similar way as before: the odd nodes have only outgoing $r$-edges and the even nodes have only incoming $r$-edges. In fact our grid structure in Figure 4 is obtained from the tree structure in Figure 1 by merging the nodes that are reachable with the same number of horizontal and vertical edges up to a certain level; that is the nodes having the same "coordinates". The key idea of our construction is that in $\mathcal{S H O \mathcal { H }} \sqcap$ it is possible to express doubly exponential counters for encoding the coordinates - a similar technique has been recently used in [2] for proving N2ExpTime-hardness of $\mathcal{S R O I} \mathcal{Q}$. We use a pair of counters to encode the coordinates of the grid: the counters are initialized in the origin $O$ of the grid; the first counter is incremented across horizontal edges and the second counter is incremented across the vertical 

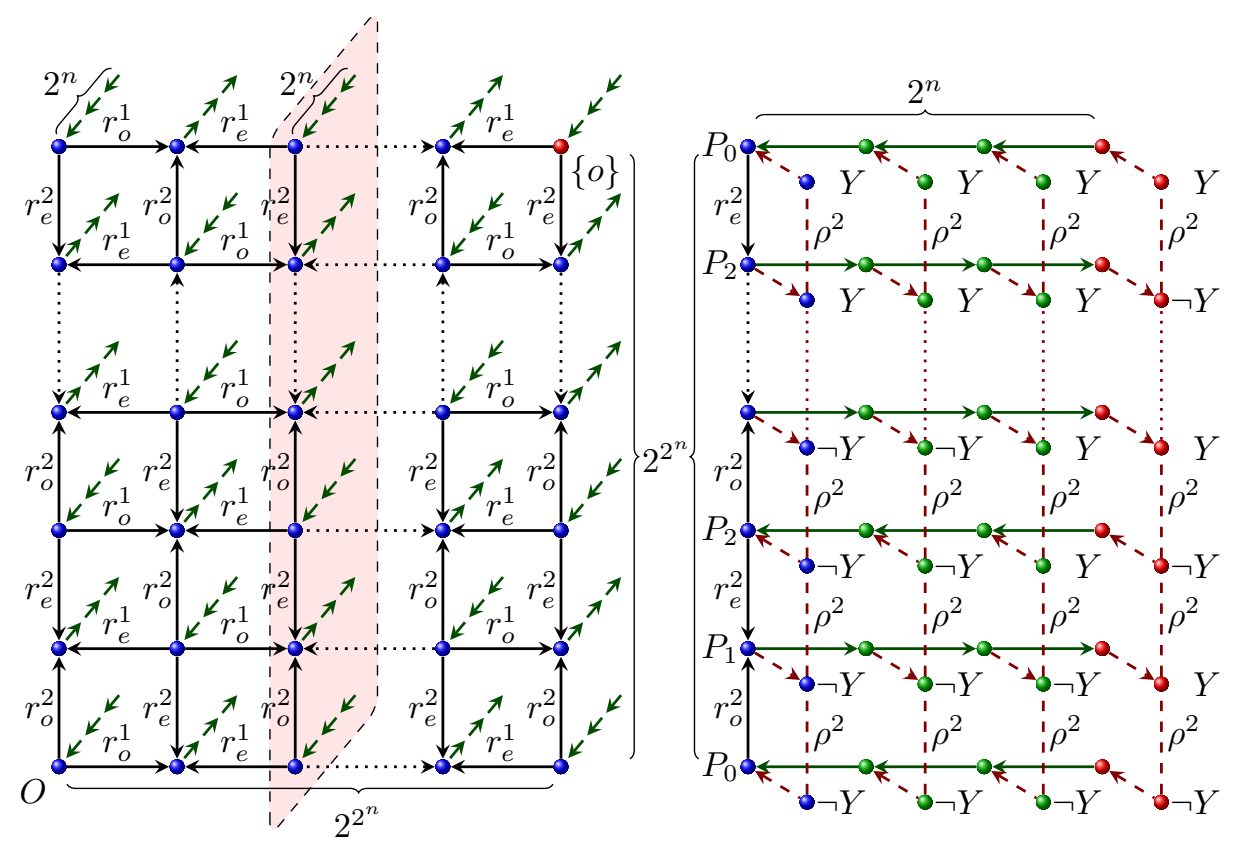

Fig. 4. A doubly exponential grid structure (left) and a detailed picture corresponding to the selected vertical slice in the grid (right)

edges. We use nominals and inverse functional roles as in the hardness prove for $\mathcal{S H O I Q}[6]$ to enforce the uniqueness of the nodes with the same coordinates.

To store the values of the counters we will use exponentially long $v$-chains that grow from the nodes of the grid. The $i^{\text {th }}$ element of the chain encodes the $i^{\text {th }}$ bit of the horizontal counter using concept $X$ and the $i^{\text {th }}$ bit of the vertical counter using concept $Y$ (see the right part of Figure 1). We will use auxiliary side $h$-chains like in our construction for $\mathcal{S H} \mathcal{H} \sqcap$ to connect the corresponding elements of the $v$-chains, which allows a proper incrementation of the counters.

In order to express the grid-like structure in Figure 4, we reuse all axioms (6)-(31) that define $r-, v$-, and $h$-chains, and add axioms to deal with the new counters and to merge the nodes with equal coordinates. First, we initialize both counters for the origin of our grid using auxiliary concepts $Z^{1}$ and $Z^{2}$ :

$$
O \sqsubseteq Z^{1} \sqcap Z^{2} \quad Z^{1} \sqsubseteq \neg X \sqcap \forall v \cdot Z^{1} \quad Z^{2} \sqsubseteq \neg Y \sqcap \forall v \cdot Z^{2}
$$

Next, we introduce two concepts $X^{f}$ and $Y^{f}$ which express that the corresponding bit of the counter needs to be flipped in the successor value. Thus, the ending bit of the counter should always be flipped, while any other bit of the counter should be flipped if and only if the lower bit of the counter (accessible via $v$ ) is 
flipped from 1 to 0 :

$$
\begin{array}{rlrl}
E & \sqsubseteq X^{f} \sqcap Y^{f} & & \\
\exists v \cdot\left(X \sqcap X^{f}\right) & \sqsubseteq X^{f} & \exists v \cdot \neg\left(X \sqcap X^{f}\right) \sqsubseteq \neg X^{f} \\
\exists v \cdot\left(Y \sqcap Y^{f}\right) & \sqsubseteq Y^{f} & \exists v . \neg\left(Y \sqcap Y^{f}\right) \sqsubseteq \neg Y^{f}
\end{array}
$$

Additionally, we express that the values of $X, Y, X^{f}$, and $Y^{f}$ agree across all elements of the same $h$-chain:

$$
\begin{aligned}
& X \sqsubseteq \forall h . X \quad \neg X \sqsubseteq \forall h . \neg X \quad Y \sqsubseteq \forall h . Y \quad \neg Y \sqsubseteq \forall h . \neg Y \\
& X^{f} \sqsubseteq \forall h . X^{f} \quad \neg X^{f} \sqsubseteq \forall h . \neg X^{f} \quad Y^{f} \sqsubseteq \forall h . Y^{f} \quad \neg Y^{f} \sqsubseteq \forall h . \neg Y^{f}
\end{aligned}
$$

Finally, we express when the bits are flipped and when they are not flipped for the successor configurations using the property that the end elements of $h$ chains are related to exactly the corresponding elements of the successor chains via the roles $\rho^{1}$ and $\rho^{2}$. The axioms are analogous to axioms (38) and (39) that propagate the information to the successor configurations:

$$
\begin{aligned}
Q_{i} \sqcap X^{f} & \sqsubseteq\left(X \sqcap \forall \rho^{1} \cdot\left[\neg Q_{[i+1]_{3}} \sqcup \neg X\right]\right) \sqcup\left(\neg X \sqcap \forall \rho^{1} \cdot\left[\neg Q_{[i+1]_{3}} \sqcup X\right]\right) \\
Q_{i} \sqcap \neg X^{f} & \sqsubseteq\left(X \sqcap \forall \rho^{1} \cdot\left[\neg Q_{[i+1]_{3}} \sqcup X\right]\right) \sqcup\left(\neg X \sqcap \forall \rho^{1} \cdot\left[\neg Q_{[i+1]_{3}} \sqcup \neg X\right]\right) \\
Q_{i} \sqcap Y^{f} & \sqsubseteq\left(Y \sqcap \forall \rho^{2} \cdot\left[\neg Q_{[i+1]_{3}} \sqcup \neg Y\right]\right) \sqcup\left(\neg Y \sqcap \forall \rho^{2} .\left[\neg Q_{[i+1]_{3}} \sqcup Y\right]\right) \\
Q_{i} \sqcap \neg Y^{f} & \sqsubseteq\left(Y \sqcap \forall \rho^{2} \cdot\left[\neg Q_{[i+1]_{3}} \sqcup Y\right]\right) \sqcup\left(\neg Y \sqcap \forall \rho^{2} .\left[\neg Q_{[i+1]_{3}} \sqcup \neg Y\right]\right)
\end{aligned}
$$

The following formulas express that the counters are copied for other directions:

$$
\begin{aligned}
& Q_{i} \sqsubseteq\left(X \sqcap \forall \rho^{2} .\left[\neg Q_{[i+1]_{3}} \sqcup X\right]\right) \sqcap\left(\neg X \sqcap \forall \rho^{2} \cdot\left[\neg Q_{[i+1]_{3}} \sqcup \neg X\right]\right) \\
& Q_{i} \sqsubseteq\left(Y \sqcap \forall \rho^{1} .\left[\neg Q_{[i+1]_{3}} \sqcup Y\right]\right) \sqcap\left(\neg Y \sqcap \forall \rho^{1} .\left[\neg Q_{[i+1]_{3}} \sqcup \neg Y\right]\right)
\end{aligned}
$$

In order to determine whether the counters have reached the maximal value $2^{2^{n}}-1$, we use concepts $E^{1}$ and $E^{2}$ that hold on the elements of $v$-chains if and only if $X$, respectively $Y$, hold for all $v$-successors until the end of the chain:

$$
\begin{array}{ll}
X \sqcap\left(E \sqcup \exists v \cdot E^{1}\right) \sqsubseteq E^{1} & E^{1} \sqsubseteq X \sqcap\left(E \sqcup \forall v \cdot E^{1}\right) \\
Y \sqcap\left(E \sqcup \exists v \cdot E^{2}\right) \sqsubseteq E^{2} & E^{1} \sqsubseteq X \sqcap\left(E \sqcup \forall v \cdot E^{1}\right)
\end{array}
$$

In order to avoid creating $r$-successors after the maximal values of the counters are reached, we replace axioms (11) and (12) with (62) and (63):

$$
\begin{aligned}
& Z \sqcap \text { Odd } \sqsubseteq\left(E^{1} \sqcup \exists r_{e}^{1} \text {.Even }\right) \sqcap\left(E^{2} \sqcup \exists r_{e}^{2} \text {.Even }\right) \\
& Z \sqcap \text { Even } \sqsubseteq\left(E^{1} \sqcup \exists r_{o}^{1^{-}} \text {.Odd }\right) \sqcap\left(E^{2} \sqcup \exists r_{o}^{2-} \text {.Odd }\right)
\end{aligned}
$$

In order to merge the elements with the same coordinates, we first merge the elements that have the maximal values for both counters:

$$
Z \sqcap E^{1} \sqcap E^{2} \sqsubseteq\{o\}
$$


The preceding elements with the same coordinates are then merged by asserting functionality of the roles $r^{1}$ and $r^{2}$ that are respective superroles of $r_{e}^{1}, r_{o}^{1^{-}}, r_{e}^{2}$, and $r_{o}^{2-}$ according to (13):

$$
\operatorname{Func}\left(r^{1}\right) \quad \operatorname{Func}\left(r^{2}\right)
$$

Our complexity result for $\mathcal{S H \mathcal { H }} \mathcal{I F}^{\square}$ is now obtained by a reduction from the bounded domino tiling problem. A domino system is a triple $D=(T, H, V)$, where $T=\{1, \ldots, k\}$ is a finite set of tiles and $H, V \subseteq T \times T$ are horizontal and vertical matching relations. A tiling of $m \times m$ for a domino system $D$ with initial condition $c^{0}=\left\langle t_{1}^{0}, \ldots, t_{n}^{0}\right\rangle, t_{i}^{0} \in T, 1 \leq i \leq n$, is a mapping $t:\{1, \ldots, m\} \times$ $\{1, \ldots, m\} \rightarrow T$ such that $\langle t(i-1, j), t(i, j)\rangle \in H, 1<i \leq m, 1 \leq j \leq m$, $\langle t(i, j-1), t(i, j)\rangle \in V, 1 \leq i \leq m, 1<j \leq m$, and $t(i, 1)=t_{i}^{0}, 1 \leq i \leq n$. It is well known [13] that there exists a domino system $D_{0}$ that is N2ExpTimecomplete for the following decision problem: given an initial condition $c^{0}$ of the size $n$, check if $D_{0}$ admits the tiling of $2^{2^{n}} \times 2^{2^{n}}$ for $c^{0}$.

In order to encode the domino problem on our grid, we use new atomic concepts $T_{1}, \ldots, T_{k}$ for the tiles of the domino system $D_{0}$. The following axioms express that every element in our structure is assigned with a unique tile and that it is not possible to have horizontal and vertical successors that do not agree with the matching relations

$$
\begin{aligned}
\top & \sqsubseteq T_{1} \sqcup \cdots \sqcup T_{k} & \\
T_{i} \sqcap T_{j} & \sqsubseteq \perp & 1 \leq i<j \leq k \\
T_{i} \sqcap \exists r^{1} \cdot T_{j} & \sqsubseteq \perp & \langle i, j\rangle \notin H \\
T_{i} \sqcap \exists r^{2} \cdot T_{j} & \sqsubseteq \perp & \langle i, j\rangle \notin V
\end{aligned}
$$

Finally, we express the initial condition of the grid:

$$
O \sqsubseteq T_{t_{1}^{0}} \sqcap \forall r^{1} .\left(T_{t_{2}^{0}} \sqcap \forall r^{1} \cdot\left(T_{t_{3}^{0}} \sqcap \forall r^{1} \cdot\left(T_{t_{4}^{0}} \sqcap \ldots \forall r^{1} \cdot T_{t_{n}^{0}} \ldots\right)\right)\right)
$$

Note that the size and the number of formulas that we have constructed is polynomial in the size of $c^{0}$. Since $D_{0}$ is fixed, we obtain a polynomial reduction from the doubly exponential domino tiling problem to the problem of $\mathcal{S H \mathcal { H } \mathcal { I }}{ }^{\sqcap}$ knowledge base satisfiability.

Theorem 3. Let $c^{0}$ be an initial condition of size $n$ for the domino system $D_{0}$ and $\mathcal{K}$ a knowledge base consisting of axioms (6)-(10), (13)-(31), and (48)(70). Then $D_{0}$ admits the tiling of $2^{2^{n}} \times 2^{2^{n}}$ for $c^{0}$ if and only if $O$ is (finitely) satisfiable in $\mathcal{K}$.

Corollary 5. The problem of (finite) concept satisfiability in the $D L \mathcal{S H O \mathcal { H }}{ }^{\square}$ is N2ExpTime-hard (and so are all the standard reasoning problems).

\section{Conclusions}

Our investigation of the computational complexity of DLs with role conjunctions is motivated by the facts that (i) role constructors recently gained attention since 
the upcoming OWL2 standard supports a much richer set of role constructors and (ii) conjunctive query answering in a DL $\mathcal{L}$ is often reducible to the knowledge base satisfiability problem for $\mathcal{L}$ with role conjunctions (e.g., for $\mathcal{S H \mathcal { L }}$ and $\mathcal{S H O Q}$ this is the case). We have shown that role conjunctions cause an exponential blowup for the DLs $\mathcal{S H} \mathcal{I}^{\sqcap}$ and $\mathcal{S H O} \mathcal{H} \mathcal{F}^{\sqcap}$. The main culprit for this are inverse roles, which we show by proving ExpTime-completeness of $\mathcal{S H} \mathcal{Q}^{\square}$. Our results imply that conjunctive query entailment for $\mathcal{S H \mathcal { I }}$ is 2 ExpTime-hard already for a bounded number of variables in the query. The previously known proof for 2ExpTime-hardness [14] has unbounded number of variables in queries.

It remains an open question whether $\mathcal{S H \mathcal { H }} \mathcal{F}^{\square}$ is N2ExpTime-complete and so far even decidability is unknown. We think that the answer to this question can shed some light on the problem of decidability of conjunctive query entailment in $\mathcal{S H O I N}$ and, thus, OWL DL, which is a long-standing open problem.

\section{References}

1. Baader, F., Calvanese, D., McGuinness, D.L., Nardi, D., Patel-Schneider, P.F., eds.: The Description Logic Handbook. Cambridge University Press (2003)

2. Kazakov, Y.: $\mathcal{R} \mathcal{I} \mathcal{Q}$ and $\mathcal{S} \mathcal{R O} \mathcal{I} \mathcal{Q}$ are harder than $\mathcal{S H O \mathcal { I }}$. In: Proc. of the 11 th Int. Conf. on the Principles of Knowledge Representation and Reasoning (KR-08), AAAI Press/The MIT Press (2008)

3. Glimm, B., Horrocks, I., Lutz, C., Sattler, U.: Conjunctive query answering for the description logic $\mathcal{S H \mathcal { I }}$. J. of Artificial Intelligence Research 31 (2008) 151-198

4. Lange, M., Lutz, C.: 2-ExpTime lower bounds for Propositional Dynamic Logics with intersection. J. of Symbolic Logic 70(5) (2005) 1072-1086

5. Glimm, B., Kazakov, Y.: Role conjunctions in expressive description logics. Technical report, University of Oxford (2008) http://www . comlab.oxford.ac.uk/ /files/361/RoleConjunctions.pdf.

6. Tobies, S.: Complexity Results and Practical Algorithms for Logics in Knowledge Representation. PhD thesis, RWTH Aachen (2001)

7. Schild, K.: A correspondence theory for terminological logics: preliminary report. In: Proc. of the 12th Int. Joint Conf. on Artificial Intelligence (IJCAI-91). (1991)

8. Kazakov, Y., Motik, B.: A resolution-based decision procedure for $\mathcal{S H O \mathcal { I }}$. J. of Automated Reasoning 40(2-3) (2008) 89-116

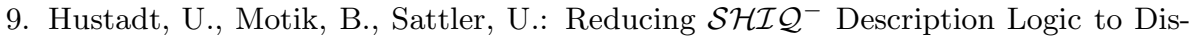
junctive Datalog Programs. In: Proc. of the 9th Int. Conf. on the Principles of Knowledge Representation and Reasoning (KR-04). (2004)

10. Sipser, M.: Introduction to the Theory of Computation, Second Edition. Course Technology (February 2005)

11. Chandra, A.K., Kozen, D.C., Stockmeyer, L.J.: Alternation. J. of the ACM 28(1) (1981) 114-133

12. Tobies, S.: The complexity of reasoning with cardinality restrictions and nominals in expressive description logics. J. of Artificial Intelligence Research 12 (2000)

13. Börger, E., Grädel, E., Gurevich, Y.: The classical decision problem. J. of Logic, Language and Information 8(4) (1999) 478-481

14. Lutz, C.: Inverse roles make conjunctive queries hard. In: Proc. of the 2007 Description Logic Workshop (DL 2007). (2007) 